University of Wollongong

Research Online

Faculty of Informatics - Papers (Archive)

Faculty of Engineering and Information

Sciences

3-10-2007

\title{
An Investigation into Temporal Gamma Luminance for Digital Fringe Fourier Transform Profilometers
}

J. M. Baker

University of Wollongong

Joe F. Chicharo

University of Wollongong, chicharo@uow.edu.au

Jiangtao Xi

University of Wollongong, jiangtao@uow.edu.au

Follow this and additional works at: https://ro.uow.edu.au/infopapers

Part of the Physical Sciences and Mathematics Commons

\section{Recommended Citation}

Baker, J. M.; Chicharo, Joe F.; and Xi, Jiangtao: An Investigation into Temporal Gamma Luminance for Digital Fringe Fourier Transform Profilometers 2007.

https://ro.uow.edu.au/infopapers/610

Research Online is the open access institutional repository for the University of Wollongong. For further information contact the UOW Library: research-pubs@uow.edu.au 


\title{
An Investigation into Temporal Gamma Luminance for Digital Fringe Fourier Transform Profilometers
}

\author{
Abstract \\ Analysis of the impact of temporal gamma luminance on Fourier Transform Profilometry (FTP) Digital \\ Video Projection (DVP) based structured light profilometers is undertaken. We investigate the spectral \\ harmonic structure for typical DVP fringe d0 images linking projector -y and 2nd order fringe harmonics. \\ The Ep validity of the presented study is verified through simulation, and subsequently we conclude that \\ for typical projector $-y$, the 2 nd order harmonic is the single most significant contribution to reconstruction \\ error for the phase measuring technique. The impact of our analysis is further gauged by empirical \\ measurement of the temporal variation of $-\mathrm{y}$ of a DVP device.

\section{Keywords} \\ Gamma, Harmonics, Non-linear Luminance, Digital, Fringe, Profilometry, FTP

\section{Disciplines} \\ Physical Sciences and Mathematics

\section{Publication Details} \\ This article was originally published as Baker, JM, Chicharo, JF and Xi, J, An Investigation into Temporal \\ Gamma Luminance for Digital Fringe Fourier Transform Profilometers, IEEE International Symposium on \\ Intelligent Signal Processing: WISP 2007, 3-5 Oct, 1-6.
}




\title{
An Investigation into Temporal Gamma Luminance for Digital Fringe Fourier Transform Profilometers
}

\author{
M. J. Baker, J. F. Chicharo and J. Xi \\ School of Electrical, Computer and Telecommunications Engineering, \\ University of Wollongong, NSW 2522, Australia \\ Tel: +6124221 3244, Fax: +61242273277 \\ E-mail: mjb06@uow.edu.au
}

\begin{abstract}
Analysis of the impact of temporal gamma luminance on Fourier Transform Profilometry (FTP) Digital Video Projection (DVP) based structured light profilometers is undertaken. We investigate the spectral harmonic structure for typical DVP fringe images linking projector $\gamma$ and 2nd order fringe harmonics. The validity of the presented study is verified through simulation, and subsequently we conclude that for typical projector $\gamma$, the 2nd order harmonic is the single most significant contribution to reconstruction error for the phase measuring technique. The impact of our analysis is further gauged by empirical measurement of the temporal variation of $\gamma$ of a DVP device.
\end{abstract}

Keywords - Gamma, Harmonics, Non-linear Luminance, Digital, Fringe, Profilometry, FTP

\section{INTRODUCTION}

Structured light techniques for non-contact, dynamic and accurate profile measurement of diffuse surfaces have been widely studied due to their potential industrial applications. The objective of our research is investigation into the application of structured light sensing technology for the potential application of 3D feature extraction of the human face. More specifically we are interested in developing an economical yet sophisticated sensor in which could be utilised to replace avators in network games with actual player features.

The most exploited techniques often utilise a projected periodic fringe pattern composed of parallel lines. The observed pattern is modulated by the diffuse surface in such a way that the modulation contains information about the height of the object perpendicular to the plane of observation. The modulated structured light pattern is recorded, commonly by a CCD camera and then processed by a fringe processing algorithm such as the popular Fourier Transform Profilometry (FTP) [1] technique to extract the spatial phase modulation. Figure 1 depicts the typical Crossed Optical Axes geometry utilised by many traditional structured light profilometers. Noting that triangle $E_{p} E_{c} D$

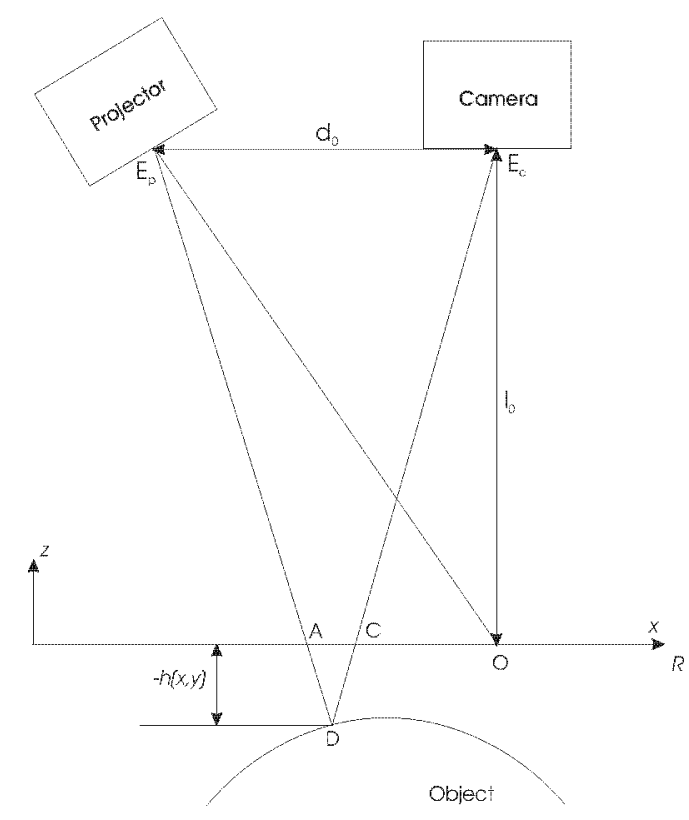

Fig. 1. TyPiCAL CROSSED OPTICAL AXES PROFILOMETRY ARRANGEMENT

and triangle $A C D$ are similar, the spatial phase modulation is thereby used to recreate the surface of interest in threedimensional space.

$$
\begin{aligned}
\frac{A C}{-h(x, y)} & =\frac{d_{0}}{l_{0}-h(x, y)} \\
h(x, y) & =\frac{l_{0} A C}{A C-d_{0}},
\end{aligned}
$$

Traditionally, structured light fringe patterns are often generated using interferometric methods via a laser source. An alternative to conventional laser projection is Digital Video 
Projection (DVP). DVP is a technology which has been actively pursued in this particular field of research as it can provide a number of key advantages. For instance, typical digital video projectors are capable of projecting standard 24 bit bitmap computer generated images and hence, when interfaced to a personal computer make a very affordable, flexible and robust projection source. However, for well exploited fringe processing algorithms such as FTP, nonlinear luminance effects commonplace with DVP significantly hinder the estimation of spatial displacement $A C$ and hence system accuracy. Commonly, the nonlinear luminance associated with DVP is referred to as Gamma distortion. Gamma distortion is typical in visual display systems to enhance human perception of the sensation of lightness, which can be regarded as a power function of intensity [2]. Gamma correction for DVP based structured light profilometers was first identified by Guo et al. in [3] whereby the iterative statistical analysis of digital fringe patterns was undertaken to correct the gamma distortion in digitally projected images. Through successful identification of projector gamma distortion Guo et al. were able to considerably reduce reconstruction errors. Alternate solutions to counter gamma non-linearities typically involve photometric fringe calibration, whereby multiple intensity distributions varying over the full range of luminance values are recorded and a camera / projector luminance curve is fitted [4], [5], however, some solutions calibrate projected fringes via other means [6], [7].

All of the proposed solutions attempt to linearise the projector I camera luminance by treating the gamma luminance nonlinearity as a static phenomenon. In fact, practically, gamma luminance presents temporal aspects, varying from fringe image to image and resulting in further reconstruction error. In this paper we analytically investigate the implications of temporal gamma and its impact on system accuracy for the well known FTP fringe processing algorithm. We subsequently verify our findings via simulation, showing that the influence of only the 2nd order harmonic component needs to be considered for the $\gamma$ modeled fringe. To further gauge the impact of the temporal gamma variation, we empirically measure the variation for a typical digital video projector, and reconstruct a known surface.

\section{PRINCIPLE FTP Fringe PROFILOMETRY}

The FTP fringe processing technique, obtains an estimate of $A C$ by processing the spatial displacement as a spatial phase displacement. The approach typically requires the projection of a sinusoidal intensity distribution (although a Ronchi grating can also be used) with spatial frequency $f_{0}$, therefore Equation (1) becomes

$$
h(x, y) \approx-\frac{l_{0}}{2 \pi f_{0} d_{0}} \Delta \phi(x, y)
$$

where

$$
\Delta \phi(x, y)=\phi(x, y)-\phi_{0}(x, y)
$$

and where $\phi(x, y)$ and $\phi_{0}(x, y)$ are the phase modulations relating to the diffuse surface to be profiled and the reference plane $R$ respectively, for the case where $l_{0} \gg h(x)$. For the sinusoidal case the following Equations represent the ideal captured fringe patterns.

$$
\begin{array}{r}
g(x, y)=a(x, y)+b(x, y) \cos \left[2 \pi f_{0} x+\phi(x, y)\right] \\
\text { and } \\
g_{0}(x, y)=a(x, y)+b(x, y) \cos \left[2 \pi f_{0} x+\phi_{0}(x, y)\right]
\end{array}
$$

where $a(x, y)$ and $b(x, y)$ are functions representing the captured fringe offset and contrast.

By the principle FTP method an estimation of phase modulation $\Delta \phi(x, y)$ can be obtained by

$$
\Delta \phi(x, y)=\operatorname{Im}\left[\log \left(\hat{g}(x, y) \hat{g}_{0}^{*}(x, y)\right)\right]
$$

where $\log (\cdot)$ denotes natural $\operatorname{logarithm}$ function, $\operatorname{Im}(\cdot)$ is the operation to acquire the imaginary part of a complex number, $\left.\hat{g}_{0}^{*}(x, y)\right)$ is the complex conjugate of $\left.\hat{g}_{0}(x, y)\right)$ the appropriately filtered reference fringe image and $\hat{g}(x, y)$ represents the appropriately filtered deformed fringe image.

Observing Equation (6) it is clear that the mathematics governing the evaluation of spatial displacement $A C$ requires the adequate generation, capture and processing of pure sinusoidal fringe images. For DVP based structured light profilometers the geometric precision of projected fringe images is often compromised due to non-linear luminance effects known as Gamma distortion. Furthermore, since the FTP method is reliant on digital filtering, inadequate filtering can result in phase residual from higher order harmonics and hence, when combining such technology it is important to understand the implications Gamma non-linearities impose.

\section{Modeling a $\gamma$ Distorted Fringe}

In general, the gamma distortion of a digital display can be modeled using the simple power function seen in Equation (7)

$$
\bar{u}(x, y)=u(x, y)^{\gamma}, \quad \text { for } u \in[0,1]
$$

where $u(x, y)$ is the normalised image delivered to the display device, $\bar{u}(x, y)$ is the actual normalised image output intensity distribution and $\gamma$ is typically a fractional value $1<\gamma<3$ specific to the display device. Considering the FTP scenario where we typically have the projection of a sinusoidal intensity distribution, Equation (7) becomes,

$$
\bar{u}(x, y)=\left[a+b \cos \left(2 \pi f_{0} x\right)\right]^{\gamma},
$$

where $f_{0}$ is the intended spatial carrier frequency of the projected fringe, and $a$ and $b$ are constants referring to the fringe offset and contrast respectively. It is clear from observation of Equation (8) the projection of a sinusoid will result in harmonics and thus we can represent a projected fringe as a Fourier Series with 
infinite $m$ th order harmonic components. Considering only the contribution of the 2 nd order harmonic we can define

$$
p=\frac{\left[a^{\gamma} \int_{0}^{T}\left[1+\frac{b}{a} \cos \left(2 \pi f_{0} x\right)\right]^{\gamma} \cos \left(4 \pi f_{0} x\right) d x\right]^{2}}{\left[a^{\gamma} \int_{0}^{T}\left[1+\frac{b}{a} \cos \left(2 \pi f_{0} x\right)\right]^{\gamma} \cos \left(2 \pi f_{0} x\right) d x\right]^{2}}
$$

the power ratio of the 2 nd order harmonic to the fundamental, which is a function of the ratio of fringe contrast and offset parameters $\frac{b}{a}$ and $\gamma$, where $T$ corresponds to the spatial period of the fringe image. Now that we have gauged the harmonic distortion of a gamma distorted sinusoid we can use Equation (9) to further evaluate the impact $\gamma$ has on system performance.

\section{STATIC $\gamma /$ HARMONIC ERROR ANALYSIS}

Assuming that $\gamma$ can be considered static, based on a previous derivation [8], we denote $\Lambda(\theta)$ as the phase measuring error associated with the 2 nd order harmonic letting $\theta$ denote $2 \pi f_{0} x+$ $\varphi(x, y)$ where $\varphi(x, y)$ represent $\phi(x, y)$ and $\phi_{0}(x, y)$ for the deformed and reference fringe respectively, and show that

$$
\begin{aligned}
\Lambda(\theta) & =\operatorname{Im}\left(\log \left(1+\sqrt{p} \cdot e^{i \theta(m-1)}\right)\right) \\
& =\arctan \left(\frac{\sqrt{p} \sin (\theta(m-1))}{1+\sqrt{p} \cos (\theta(m-1))}\right)
\end{aligned}
$$

where clearly $m=2$. It can be further shown that the maximum absolute phase measuring error can be given as

$$
\varepsilon_{\max }=2 \arctan \left(\sqrt{\frac{p}{1-p}}\right)
$$

and hence using Equation (2), the maximum absolute height measurement error $\beta$ can be given as

$$
\beta=\frac{l_{0}}{\pi f_{0} d_{0}} \cdot \arctan \left(\sqrt{\frac{p}{1-p}}\right)
$$

If we now assume system parameters $l_{0}, d_{0}$ and $f_{0}$ are $5 \mathrm{~m}$, $2 \mathrm{~m}$ and $10 / \mathrm{m}$ respectively, we can plot a curve describing the relationship, as depicted in Figure (2). It can be seen that for larger values of $\gamma$ i.e. stronger harmonics, the greater the profile measurement error. It is important to note that this derivation does not take filtering into consideration and therefore, provides insight into the worst case scenario only. Nevertheless, the influence of filtering can simply be likened to reducing the magnitude of the $\gamma$ term.

\section{TEMPORAL $\gamma /$ HARMONIC ERROR ANALYSIS}

The above expressions describe the errors associated with $\gamma$ when it can be considered static. In practice it has been observed that the harmonic spectral distribution presents temporal aspects

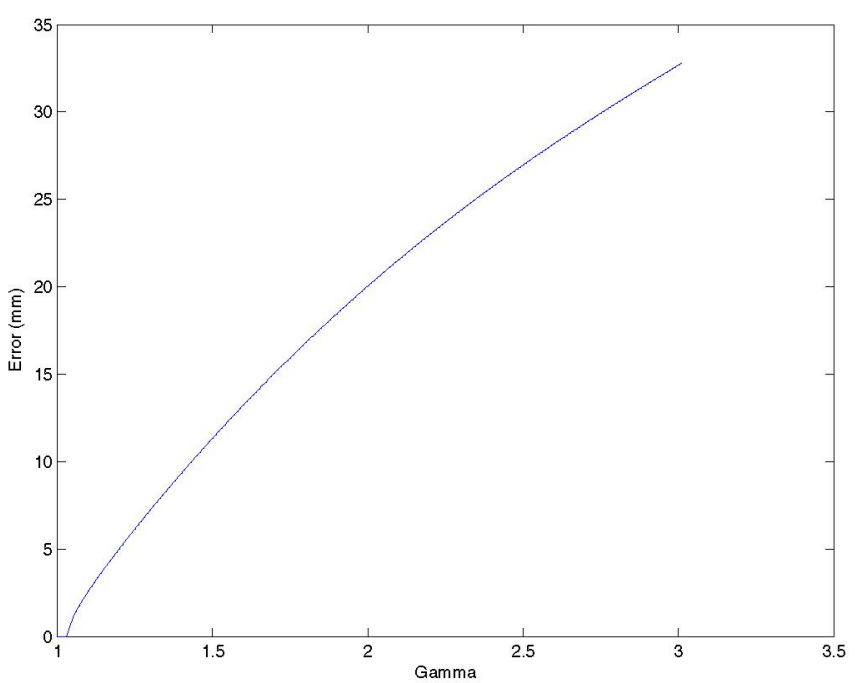

Fig. 2. MAXIMUM ABSOLUTE PROFILE MEASUREMENT ERROR: FOR FRINGE PARAMETERS $a=b$

and hence further consideration of this analysis needs to be undertaken to measure the influence of this phenomenon.

If we consider $\gamma$ and $\gamma_{0}$, the gamma values for both the deformed and reference images respectively, we can define the captured $\gamma$ distorted fringe images as

$$
\begin{array}{r}
\bar{g}(x, y)=a(x, y)+b(x, y) \cos \left[2 \pi f_{0} x+\phi(x, y)\right] \\
+c_{m}(x, y) \cos \left(m\left[2 \pi f_{0} x+\phi(x, y)\right]\right)
\end{array}
$$

and

$$
\begin{array}{r}
\bar{g}_{0}(x, y)=a(x, y)+b(x, y) \cos \left[2 \pi f_{0} x+\phi_{0}(x, y)\right] \\
+c_{0 m}(x, y) \cos \left(m\left[2 \pi f_{0} x+\phi_{0}(x, y)\right]\right)
\end{array}
$$

where $c_{m}(x, y)$ and $c_{0 m}(x, y)$ are the functions representing the $m$-th order harmonic component for the deformed and reference fringe respectively.

Using the FTP method, we can obtain $\Delta \hat{\phi}(x, y)$ an estimation of the phase map $\Delta \phi(x, y)$ by

$$
\Delta \hat{\phi}(x, y)=\operatorname{Im}\left(\log \left(\bar{g}(x, y){\overline{g_{0}}}^{*}(x, y)\right)\right)
$$

Discarding the de terms and substituting Equations (13) and (14) it can be seen that

$$
\begin{aligned}
\Delta \hat{\phi}= & \operatorname{Im}\left(\log \left(b \cdot e^{i\left(2 \pi f_{0} x+\phi\right)}+c_{m} \cdot e^{i\left(2 \pi f_{0} x+\phi\right) m}\right)\right. \\
& \left.+\log \left(b \cdot e^{-i\left(2 \pi f_{0} x+\phi_{0}\right)}+c_{0 m} \cdot e^{-i\left(2 \pi f_{0} x+\phi_{0}\right) m}\right)\right) \\
= & \operatorname{Im}\left(\log \left(e^{i\left(2 \pi f_{0} x+\phi\right)}+\sqrt{p} \cdot e^{i\left(2 \pi f_{0} x+\phi\right) m}\right)\right. \\
& \left.+\log \left(e^{-i\left(2 \pi f_{0} x+\phi_{0}\right)}+\sqrt{p_{0}} \cdot e^{-i\left(2 \pi f_{0} x+\phi_{0}\right) m}\right)\right) \\
= & \operatorname{Im}\left(i\left(2 \pi f_{0} x+\phi\right)+\log \left(1+\sqrt{p} \cdot e^{i\left(2 \pi f_{0} x+\phi\right)(m-1)}\right)\right. \\
& \left.-i\left(2 \pi f_{0} x+\phi_{0}\right)+\log \left(1+\sqrt{p_{0}} \cdot e^{-i\left(2 \pi f_{0} x+\phi_{0}\right)(m-1)}\right)\right)
\end{aligned}
$$




$$
\begin{aligned}
= & \operatorname{Im}\left(i \phi+\log \left(1+\sqrt{p} \cdot e^{i\left(2 \pi f_{0} x+\phi\right)(m-1)}\right)\right. \\
& \left.+\log \left(1+\sqrt{p_{0}} \cdot e^{-i\left(2 \pi f_{0} x+\phi_{0}\right)(m-1)}\right)\right) \\
= & \Delta \phi+\operatorname{Im}\left(\log \left(1+\sqrt{p} \cdot e^{i\left(2 \pi f_{0} x+\phi\right)(m-1)}\right)\right. \\
& \left.+\log \left(1+\sqrt{p_{0}} \cdot e^{-i\left(2 \pi f_{0} x+\phi_{0}\right)(m-1)}\right)\right)
\end{aligned}
$$

where $p=\frac{c_{m}^{2}}{b^{2}}$ and $p_{0}=\frac{c_{0 m}^{2}}{b^{2}}$ represent the power ratio of the $m$ th harmonic to the fundamental, for the deformed and reference fringe images.

Hence, the estimation error of the phase can be expressed as:

$$
\begin{aligned}
\varepsilon= & \Delta \hat{\phi}-\Delta \phi \\
= & \Delta \phi+\operatorname{Im}\left(\log \left(1+\sqrt{p} \cdot e^{i\left(2 \pi f_{0} x+\phi\right)(m-1)}\right)\right. \\
& \left.+\log \left(1+\sqrt{p_{0}} \cdot e^{-i\left(2 \pi f_{0} x+\phi_{0}\right)(m-1)}\right)\right)-\Delta \phi \\
= & \operatorname{Im}\left(\log \left(1+\sqrt{p} \cdot e^{i\left(2 \pi f_{0} x+\phi\right)(m-1)}\right)\right. \\
& \left.+\log \left(1+\sqrt{p_{0}} \cdot e^{-i\left(2 \pi f_{0} x+\phi_{0}\right)(m-1)}\right)\right)
\end{aligned}
$$

Now defining $\Lambda(\cdot)$, the function to extract the phase angle of the complex number, we can now show

$$
\begin{aligned}
\Lambda(\theta, \rho) & =\operatorname{Im}\left(\log \left(1+\sqrt{\rho} \cdot e^{i \theta(m-1)}\right)\right) \\
& =\arctan \left(\frac{\sqrt{\rho} \sin (\theta(m-1))}{1+\sqrt{\rho} \cos (\theta(m-1))}\right)
\end{aligned}
$$

where we set $m=2$ for the second order contribution and $\rho$ represents the respective fringe power ratios.

Now rewriting Equation (17), the phase measuring error can be given as

$$
\begin{aligned}
\varepsilon & =\Lambda\left(2 \pi f_{0} x+\phi, p\right)+\Lambda\left(-\left(2 \pi f_{0} x+\phi_{0}, p_{0}\right)\right) \\
& =\Lambda\left(2 \pi f_{0} x+\phi, p\right)-\Lambda\left(2 \pi f_{0} x+\phi_{0}, p_{0}\right)
\end{aligned}
$$

And furthermore, we can define a maximum measurement error

$$
\begin{aligned}
\varepsilon_{\max } & =\Lambda_{\max }\left(2 \pi f_{0} x+\phi, p\right)-\Lambda_{\min }\left(2 \pi f_{0} x+\phi_{0}, p_{0}\right) \\
& =\Lambda_{\max }(\theta, p)-\Lambda_{\min }\left(\theta, p_{0}\right)
\end{aligned}
$$

By letting $\frac{\mathrm{d} \Lambda}{\mathrm{d} \theta}=0$, we can derive the maximum and minimum value of $\Lambda$ respectively. That is

$$
\Lambda_{\max }=\arctan \left(\sqrt{\frac{p}{1-p}}\right)
$$

and

$$
\Lambda_{\text {min }}=-\arctan \left(\sqrt{\frac{p_{0}}{1-p_{0}}}\right)
$$

Equation(20) can then be expressed as

$$
\varepsilon_{\max }=\arctan \left(\sqrt{\frac{p}{1-p}}\right)+\arctan \left(\sqrt{\frac{p_{0}}{1-p_{0}}}\right)
$$

and hence substituting Equation (23) into Equation (2), the (16) maximum absolute height measurement error $\beta$ is given as

$$
\beta=\frac{l_{0}}{2 \pi f_{0} d_{0}} \cdot\left[\arctan \left(\sqrt{\frac{p}{1-p}}\right)+\arctan \left(\sqrt{\frac{p_{0}}{1-p_{0}}}\right)\right]
$$

\section{A. Gamma Compensated Analysis}

Since we now have an expression to describe the measurement error, the obvious question is what impact does the temporal variation of $\gamma$ have on a $\gamma$ compensated system. Clearly, this would be a factor dependent on the way in which $\gamma$ was compensated and also the amount of temporal variation. For simplicity if we assume that just one of the fringe images had been appropriately compensated we can gauge the phase measuring error as

$$
\varepsilon=\Lambda\left(2 \pi f_{0} x+\phi, \rho\right)
$$

and hence the maximum measurment error as

$$
\beta=\frac{l_{0}}{2 \pi f_{0} d_{0}} \cdot \arctan \left(\sqrt{\frac{\rho}{1-\rho}}\right)
$$

where $\rho$ represents the temporal difference between the two $\gamma$ compensated fringe images.

\section{Simulation}

In order to verify the validity of the analytical study we simulated a range of digitally projected fringe images with various values of $\gamma$ and $\gamma_{0}$ and measured the corresponding system accuracy for the FTP reconstruction of a simulated surface. System parameters $l_{0}, d_{0}$ and $f_{0}$ were chosen to be $5 \mathrm{~m}$, $2 \mathrm{~m}$ and $10 / \mathrm{m}$ respectively, corresponding to a spatial period of $100 \mathrm{~mm}$ if we assume an image spatial resolution of 1pixel $/ \mathrm{mm}$. The simulated fringe images are given as

$$
g(x, y)=\left[0.5+0.5 \cos \left(2 \pi f_{0} x+\phi(x, y)\right),\right]^{\gamma},
$$

and

$$
g_{0}(x, y)=\left[0.5+0.5 \cos \left(2 \pi f_{0} x+\phi_{0}(x, y)\right),\right]^{\gamma_{0}},
$$

where $\phi(x, y)$ corresponds to the hemispherical dome shape seen in Figure $4(\mathrm{a})$. $\gamma_{0}$ was varied by $+/-5 \%$ of $\gamma$ and based on the system parameters we plot the expected maximum absolute error $\beta$, according to the analytical study and also measure the reconstruction error resulting from the simulated gamma distorted fringes. We reframed from filtering in our FTP simulation apart from the necessary $\mathrm{dc}$ and negative frequency components to reveal the full influence of higher order harmonics. The results are displayed in Figure (3). The 


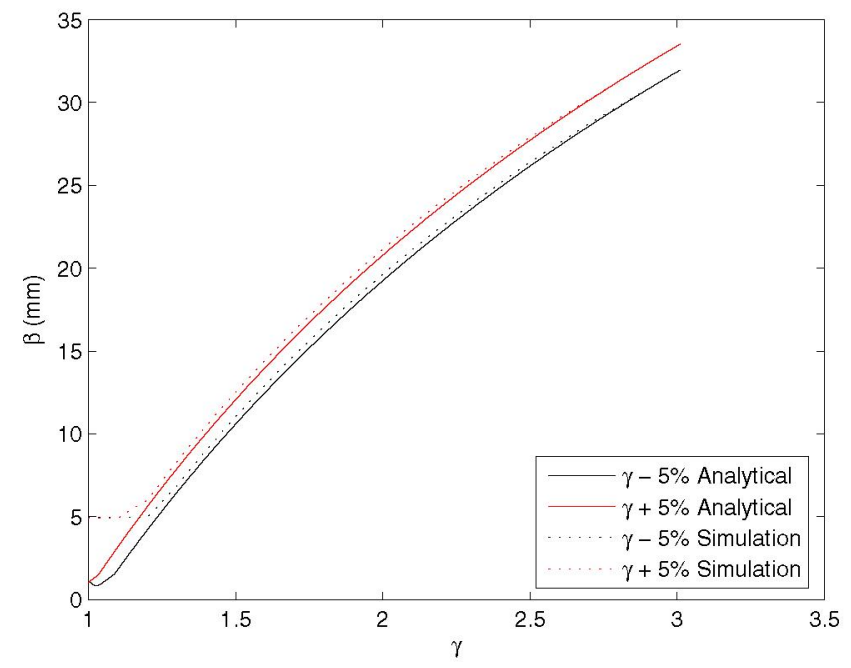

Fig. 3. MAXIMum ABSOLUTE PROFILE MEASUREMENT ERROR: $\gamma+/-5 \%$

analytical error curves are represented with the solid line, with the simulation results indicated with the dotted lines.

As can be seen the analytical derivation accurately models the maximum absolute errors resulting from fringe temporal gamma distortion. In fact, it can be seen even as $\gamma \rightarrow 3$ and the magnitude of the higher order harmonics increase, there is little if any deviation between the analytical and FTP simulated curves. The only major discrepancy is seen for the very low values of gamma. This discrepancy is directly a result of the inherent accuracy limitations of the fft operation involved in FTP reconstruction.

Based on these findings we have verified the analytical study describing the relationship between temporal projector gamma and reconstruction error for the FTP fringe processing approach. Furthermore, we conclude that for typical projector $\gamma$ fringe modeling, the 2 nd order harmonic component is the single most significant contribution to phase measuring error for the FTP technique.

\section{A. Example FTP Reconstruction}

To further gauge the significance of this study we measure the variance in $\gamma$ for a typical DLP projector. Since DLP projectors temporally project RGB components, the temporal effects of $\gamma$ are easily observed, as each colour channel typically yields an individual $\gamma$ characteristic. We project a fringe image using an InFocus LP530 DLP projector onto a reference plane from a distance of approximately $1500 \mathrm{~mm}$. We capture the projected fringe image using a DuncanTech MS-3100 3-CCD camera. The integration time of the camera was adjusted to be approximately $20 \mathrm{~ms}$ and a number of images of the projected fringe were obtained and the magnitude of $p$ was measured. The variation in $p$ is shown in Figure 5.

Based on the data obtained and displayed in Figure 5, using Equation 9 we measured a maximum $\gamma$ value of 2.47 and a

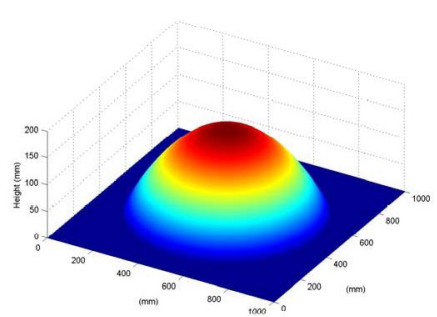

(a) Simulated Height Distribution

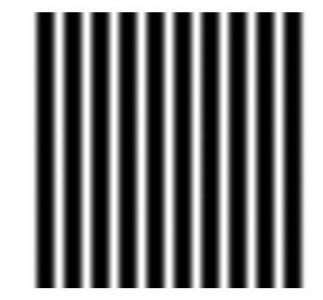

(b) Reference Fringe, $\gamma_{0}=$ 2.47

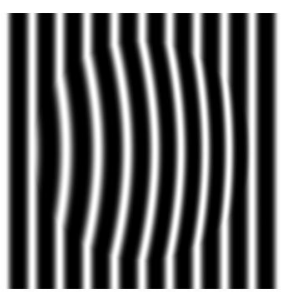

(c) Deformed Fringe, $\gamma=2.13$

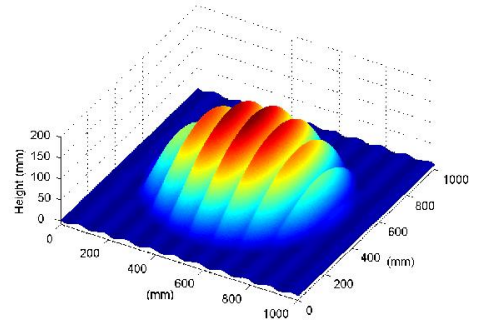

(d) FTP Reconstructed Surface
Fig. 4. Simulated Reconstruction

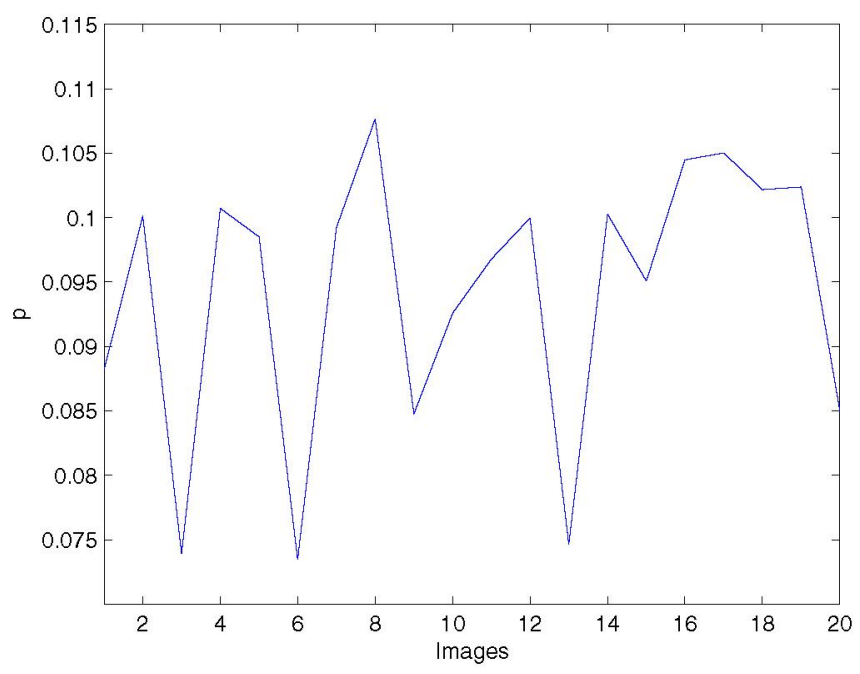

Fig. 5. TEMPORAL VARIATION IN $p$

minimum of 2.13. Using these two values we simulated the reconstruction of the diffuse surface seen in Figure 4(a) with system parameters $l_{0}, d_{0}$ and $f_{0}$ of $1500 \mathrm{~mm}, 400 \mathrm{~mm}$ and $10 / \mathrm{m}$ respectively, where 1 pixel $=1 \mathrm{~mm}$. The simulated projected fringes can be seen as Figures 4(b) and (c) respectively, with the reconstructed surface shown as Figure 4(d). As can be seen the harmonic distortion significantly reduces the accuracy of the FTP method. The mean measurement error and standard deviation was found to be $8.207 \mathrm{~mm}$ and $5.191 \mathrm{~mm}$ respectively. The temporal variation of $\gamma$ is distinctly observable in the rippled reconstruction of the reference plane surface around the 


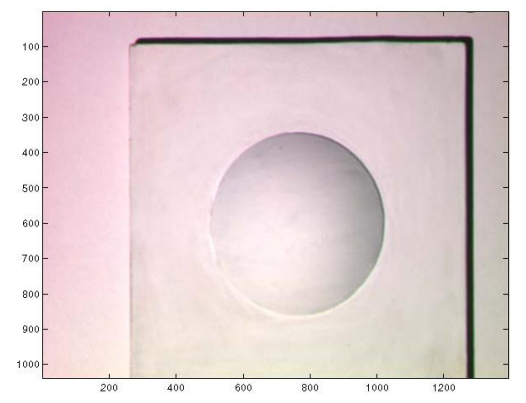

(a) Experimental Surface

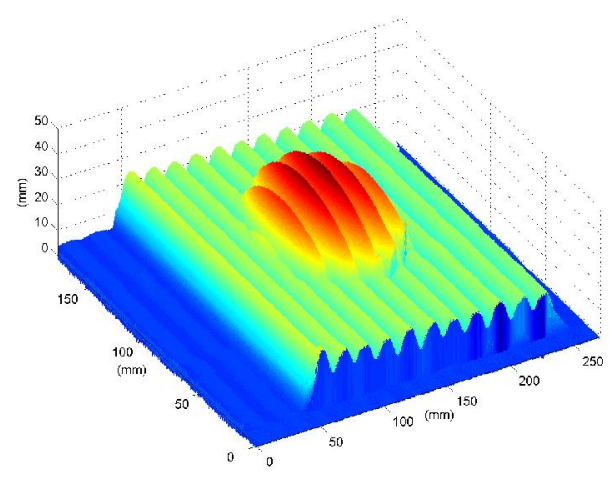

(b) FTP Reconstruction of diffuse surface

Fig. 6. EMPIRICAL FTP RECONSTRUCTION

outer edges of the surface. We will now show this rippling is commonplace in our experimental findings.

\section{EMPIRICAL RECONSTRUCTION}

To clearly demonstrate the rippling effect associated with the temporal spectral characteristics of the projected fringe distributions, we experimentally measure a simple surface using the FTP method. The spatial resolution of the captured fringe image was measured to be $0.194 \mathrm{~mm} /$ pixel, yielding a spatial period of $17.1 \mathrm{~mm}$ equating to a spatial frequency $f_{0}$ of 58.5 fringes $/ \mathrm{m}$. System parameters $l_{0}$ and $d_{0}$ were measured to be approximately $1.405 \mathrm{~m}$ and $0.465 \mathrm{~m}$ respectively. The profiled surface was a convex dome shape as seen in Figure 6(a). The maximum height of the hemispherical surface is $22.8 \mathrm{~mm}$ with a diameter of $99 \mathrm{~mm}$ with the thickness of the base material being $16 \mathrm{~mm}$. Fringe offset and contrast parameter were set such that $a=b$ for maximum harmonic distortion. The FTP reconstructed surface is shown as Figure 6(b). As can be seen the rippling effect is quite evident for the modulated parts of the image and also for the reference plane surface. This rippling of the reference plane reconstruction clearly distinguishes the temporal nature of the projected images.

\section{CONCLUSION}

In this paper we have investigated the influence of temporal $\gamma$ luminance for DVP based FTP's. The harmonic structure of a digitally projected fringe was investigated and consequently we derived an expression to link temporal harmonic distortion and phase measuring error for the FTP method. The validity of the analytical derivation was confirmed and it was concluded that for typical projector $\gamma$, the 2 nd order harmonic is the single most significant contribution to reconstruction error for the phase measuring technique. With more and more industrial applications utlising digital technology in profilometric sensing, derivations such as that presented in this paper are essential to improve the understanding of the limitations and system accuracy of the FTP phase measuring algorithm.

\section{REFERENCES}

[1] X. Su, W. Chen, "Fourier transform profilometry: a review," Optics and Lasers in Engineering, vol. 35, pp. 263-284, 2001.

[2] C. A. Poynton, "Gamma and it disguises: The nonlinear mappings of intensity in perception, crts, film and video," SMPTE Joumal, pp. 1099$1108,1993$.

[3] H. Guo, H. He, M. Chen, "Gamma correction for digital fringe projection profilometry," Applied Optics, vol. 43, no. 14, pp. 2906 - 2914, May 2004.

[4] L. Kinell, "Multichannel method for absolute shape measurement using projected fringes," Optics and Lasers in Engineering, vol. 41, pp. 57-71, 2004.

[5] P. S. Huang, Q. Hu, F. Jin, F. Chiang, "Color-encoded digital fringe projection technique for high speed three-dimensional surface contouring," Optical Engineering, vol. 38, pp. 1065-1071, 1999.

[6] M. J. Baker, J. Xi, J. F. Chicharo, "Neural network digital fringe calibration technique for structure light profilometers," Applied Optics, vol. 46, no. 8, pp. 1233-1243, March 2007.

[7] H. Zhi, R. B. Johansson, "Adaptive filter for enhancement of fringe patterns," Optics and Lasers in Engineering, vol. 15, pp. 241-251, 1991.

[8] Y. Hu, J. Xi, E. Li, J. Chicharo, Z. Yang. "Three-dimensional profilometry based on shift estimation of projected fringe patterns," Applied Optics, vol. 45 , no. 4 , pp. $678-687$, Feburary 2006 . 